\title{
USE AND LIMITATIONS OF THE HARMONIC BALANCE METHOD FOR RUB-IMPACT PHENOMENA IN ROTOR-STATOR DYNAMICS
}

\author{
Loïc Peletan* \\ Sebastien Baguet \\ Georges Jacquet-Richardet \\ University of Lyon, CNRS, INSA Lyon, LaMCoS UMR5259 \\ Villeurbanne F-69621 \\ France \\ Email: loic.peletan@insa-lyon.fr \\ Email: sebastien.baguet@insa-lyon.fr \\ Email: georges.jacquet@insa-lyon.fr
}

\author{
Mohamed Torkhani \\ LaMSID \\ Électricité de France R\&D (EDF R\&D) \\ Clamart, 92140 \\ France \\ mohamed.torkhani@edf.fr
}

\begin{abstract}
In the present paper, a Harmonic Balance Method (HBM) coupled with a pseudo-arc length continuation algorithm is presented for the prediction of the steady state behaviour of a rotorstator contact problem. The ability of the HBM to reproduce the four most common phenomena encountered during rotor to stator contact situations (i.e. 'no-rub', 'full annular rub', 'partial rub' and 'backward whirl/whip') is investigated. A modified Jeffcott rotor model is used and results of the proposed algorithm are compared with traditional time marching solutions and analytical predictions. The advantages and limitations of the HBM for this kind of problem are analyzed. It is shown that the HBM is orders of magnitude faster than transient simulations, and provides very accurate results. However, in its current form it is unable to predict quasi-periodic behaviour. Detailed analysis of the transient solutions yields valuable information for the future extension of the HBM to efficient quasi-periodic simulations.
\end{abstract}

\section{INTRODUCTION}

One of the objectives of turbomachinery designers is to improve the machines efficiency. This can be achieved by reducing the operating gap between the rotating and stationary parts of the rotor, but leads to an increased risk of rotor to stator con- tact. For safety reasons, designers must ensure that such interactions cannot have serious consequences. When an accurate prediction of the system response is required, it is insufficient to consider the linear response of the system alone. When nonlinearities are considered, highly complex dynamic behaviours are revealed, even for very simple rotor models. The simplest rotor model encountered in the literature is the Jeffcott rotor. Although this model has only one node, with two degrees of translational freedom (one for each radial direction), it may exhibit a rich variety of phenomena, due to the presence of nonlinearities. Edwards [1] studied a Jeffcott rotor with an additional degree of torsional freedom, when subjected to a mass imbalance and contact with a circular, rigid, fixed stator. The contact was modelled using a penalty method with a Coulomb friction law. The system of equations was solved by numerical integration (Runge-Kutta). Edwards showed that even when the rotor is excited by a periodic force (i.e. an imbalance force) only, this relatively simple system can exhibit periodic as well as chaotic forms of behaviour.

Many authors have studied nonlinear rotor models, using time marching techniques to reach the steady state response of the system [1-5]. This approach, although essential when investigating the system's transient behaviour $[2,6,7]$, is not optimal in terms of CPU time when the steady state response is investigated, because many cycles need to be simulated before the transient behaviour disappears completely. This can be problematic 
when parametric studies are carried out and/or when more complex models (such as finite element rotor models [4, 8]) are used.

On the other hand, some authors such as Jiang $[9,10]$ or Bently et al. [11] have used mathematical tools to study a Jeffcott rotor, for which they analytically determined the limits of existence for different types of behaviour, according to a set of dimensionless parameters such as the dimensionless rotational speed of the rotor or the coefficient of friction. Periodic and quasi-periodic phenomena were analyzed, and the periodic phenomena were found to be: 'no-rub motion', in which the rotor eccentricity is smaller than the operating gap, and 'full annular rub', in which the rotor is in permanent contact with the stator in a forward whirl mode, with a constant eccentricity. The quasiperiodic phenomena are 'partial rub', in which the rotor comes into intermittent contact with the stator, and self-excited 'backward whip' or 'backward whirl', in which the rotor rolls (respectively with or without sliding) on the inner surface of the stator, in a reverse whirl motion, with large eccentricities and large contact forces. The latter phenomenon is known to be quite violent and destructive. The four aforementioned phenomena are the most commonly reported in rotor-to-stator contact scenarios. These analytical methods are appealing, because they prove that several stable solutions can coexist with a given set of parameters. For instance, in [10] Jiang shows that a Jeffcott rotor can, for a given set of parameters and a certain range of rotational speeds, be in periodic full annular rub, as well as quasi-periodic backward whip. Time integration methods would lead to only one of these two solutions, depending on the initial conditions. These analytical methods have been extended to multi-mode rotor models by Childs and Bhattacharya [12]. Their predictions appear to be in good agreement with numerical simulations and experiments. Nevertheless, these analytical methods require non-trivial mathematical developments, and further extensions are still needed before they can be used to analyse more complex models.

Alternatively, although widely used to solve other types of problems, numerical techniques such as shooting or harmonic balance methods are rarely used for rotor-to-stator contact problems. Although such techniques do not require transient behaviour simulations, and are in practice much faster, they are limited to the study of periodic movements and, to a certain extent, to quasi-periodic movements. This limitation to periodic movements is the reason for which such techniques are generally limited to the study of geometric nonlinearities or oil-film types of mounting nonlinearities (such as in [13]), because it is known that with this kind of nonlinearity the system response remains periodic. Nevertheless, for rotor-stator contact problems coupled with a path-following continuation scheme and stability analysis, these methods prove to be helpful in parametric studies, thus minimising the use of long transient simulations.

Following a brief presentation of the harmonic balance method, a Jeffcott rotor model described in [10] is studied, and the numerical results provided by this technique are compared to
Jiang's predictions, as well as to the solutions given by traditional time marching techniques. The ability of the HBM to reproduce the four aforementioned phenomena is studied, for several values of the dry friction coefficient $\mu$. The advantages and limitations of the proposed method are analyzed. The simulated transient behaviour of the rotor is then analysed spectrally, and valuable results are derived for a future extension of this HBM to quasiperiodic situations.

\section{HARMONIC BALANCE METHOD (HBM)}

The Harmonic Balance Method (HBM) is a well-known technique for the computation of periodic solutions for dynamic systems. This method consists in solving the equations of motion in the frequency domain, rather than in the time domain. The general time-domain equation for a dynamic mechanical system is given by (1).

$$
R(q(t))=K q(t)+C \dot{q}(t)+M \ddot{q}(t)+f(q(t), \dot{q}(t))-p(t)=0
$$

where $q$ represents the displacement vector for all degrees of freedom (DOFs); $K, C$ and $M$ are the generalized stiffness, damping and mass matrices; $f$ is the nonlinear force vector and $p$ is the external excitation force vector.

When the external excitations are periodic, it can be assumed that a steady state solution exists for (1), and that this solution is also periodic. The displacements can thus be written as a truncated Fourier series:

$$
q(t)=Q_{0}+\sum_{j=1}^{N}\left(Q_{2 j-1} \cos \left(m_{j} \omega t\right)+Q_{2 j} \sin \left(m_{j} \omega t\right)\right)
$$

where $Q_{i}, \quad i \in[1 \ldots N]$ denote the Fourier coefficients of $q$; $\omega$ is the fundamental frequency of the external excitation, and $m_{i}$, for $i \in[1 \ldots N]$, are positive integers.

Similarly, $f(q)$ and $p(t)$ can be rewritten using the same form:

$$
\begin{aligned}
& p(t)=P_{0}+\sum_{j=1}^{N}\left(P_{2 j-1} \cos \left(m_{j} \omega t\right)+P_{2 j} \sin \left(m_{j} \omega t\right)\right) \\
& f(t)=F_{0}+\sum_{j=1}^{N}\left(F_{2 j-1} \cos \left(m_{j} \omega t\right)+F_{2 j} \sin \left(m_{j} \omega t\right)\right)
\end{aligned}
$$

As described in [14], equations (2) (3) and (4) are substituted into (1), and a Galerkin procedure is applied to obtain the following algebraic system:

$$
R(Q, \omega)=Z(\omega) Q+F(Q)-P=0
$$


where:

$$
\begin{array}{r}
Z=\operatorname{diag}\left(K,\left[\begin{array}{cc}
K-m_{1}^{2} \omega^{2} M & m_{1} \omega C \\
-m_{1} \omega C & K-m_{1}^{2} \omega^{2} M
\end{array}\right], \ldots,\right. \\
\left.\left[\begin{array}{cc}
K-m_{N}^{2} \omega^{2} M & m_{N} \omega C \\
-m_{N} \omega C & K-m_{N}^{2} \omega^{2} M
\end{array}\right]\right)
\end{array}
$$

$Q=\left[Q_{0}^{T}, Q_{1}^{T}, \ldots, Q_{2 N}^{T}\right]^{T}, \quad F=\left[F_{0}^{T}, F_{1}^{T}, \ldots, F_{2 N}^{T}\right]^{T}$ and $P=\left[P_{0}^{T}, P_{1}^{T}, \ldots, P_{2 N}^{T}\right]^{T}$ are the Fourier coefficient vectors for displacements, nonlinear forces and external excitations, respectively.

Equation (5) needs to be solved for $Q$. This equation is still nonlinear, such that an appropriate nonlinear solver should be used to determine the correct solution. A Newton-Raphson solver was used in the present case, which solves (5) in an iterative manner:

$$
Q^{(k+1)}=Q^{(k)}+\left(\frac{\partial R\left(Q^{(k)}\right)}{\partial Q}\right)^{-1} R\left(Q^{(k)}\right)
$$

where $R\left(Q^{(k)}, \omega\right)=Z(\omega) Q^{(k)}+F\left(Q^{(k)}\right)-P$ is the residual of equation (5). It has been shown that the combination of a HBM and a Newton-Raphson solver is equivalent to the Incremental Harmonic Balance Method (IHBM) [15]. As the relationship between the displacements and the nonlinear forces is known in the time domain only (a priori unknown in the frequency domain (5)), an Alternating Frequency Time (AFT) algorithm [16] was used for the computation of $F(Q)$. Direct and inverse Fourier transforms must be used in order to determine the solution. Displacements $(Q)$ in the frequency domain are transferred to the time domain using an inverse Fourier transform. Once the displacements have been determined in the time domain $(q)$, the corresponding nonlinear forces are calculated, following which a direct Fourier transformation is used to obtain the nonlinear forces in the frequency domain $(F)$. The next Newton-Raphson iteration can then be carried out. In practice, fast Fourier transform (FFT) algorithms are used due to their combined speed, accuracy and robustness. This procedure can be summarized as follows:

$$
Q(\omega) \stackrel{f f t^{-1}}{\longrightarrow} q(t) \longrightarrow f(t) \stackrel{f f t}{\longrightarrow} F(\omega)
$$

Similarly, for reasons of improved accuracy and faster convergence, the Jacobian term $\frac{\partial R\left(Q^{(k)}\right)}{\partial Q}$ is calculated using analytical derivation rather than finite differences.

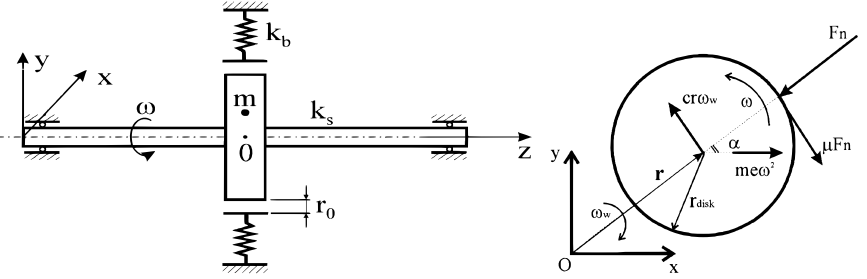

FIGURE 1. JIANG'S SCHEMATIC DIAGRAMS OF THE JEFFCOTT ROTOR WITH A CIRCULAR RIGID STATOR [10].

The path following technique used for the parametric study was a pseudo-arc length continuation method. Contrary to transient time marching techniques, the combination of HBM and a continuation method converges to both stable and unstable solutions, and there is no way to make an a priori assessment of the solution's stability. Several definitions of the stability of a dynamic solution exist. In the present study, we investigate the local stability of the solutions. The local stability assessment consists in applying a small perturbation to the equilibrium solution, and then verifying that this perturbation subsides over time. The stability can be computed a posteriori, using different theories. One theory frequently cited in the literature is that of Floquet. In the following, the stability of the HBM solutions is calculated in the time domain, by means of a monodromy matrix computation. The monodromy matrix is a linear operator describing the evolution over one period of perturbations, applied to the periodic solution. Its eigenvalues are called the Floquet multipliers. If any of the Floquet multipliers has a module greater than one, the solution is unstable, otherwise it is stable.

\section{STUDY OF A NONLINEAR JEFFCOTT ROTOR Description of the problem}

In this section, a nonlinear Jeffcott rotor identical to that described by Jiang in [10] is studied (see Fig. 1). The mathematical model has two degrees of freedom and the dimensionless equations of motion are as follows:

$$
\begin{array}{r}
X^{\prime \prime}+2 \xi X^{\prime}+\beta X+\Theta\left(1-\frac{R_{0}}{R}\right)\left(X-\mu \operatorname{sign}\left(V_{\text {rel }}\right) Y\right) \\
=\Omega^{2} \cos \Omega \tau
\end{array}
$$

$$
\begin{array}{r}
Y^{\prime \prime}+2 \xi Y^{\prime}+\beta Y+\Theta\left(1-\frac{R_{0}}{R}\right)\left(Y+\mu \operatorname{sign}\left(V_{\text {rel }}\right) X\right) \\
=\Omega^{2} \sin \Omega \tau
\end{array}
$$

$$
V_{\text {rel }}=R_{\text {disk }} \Omega+R \omega_{b}
$$




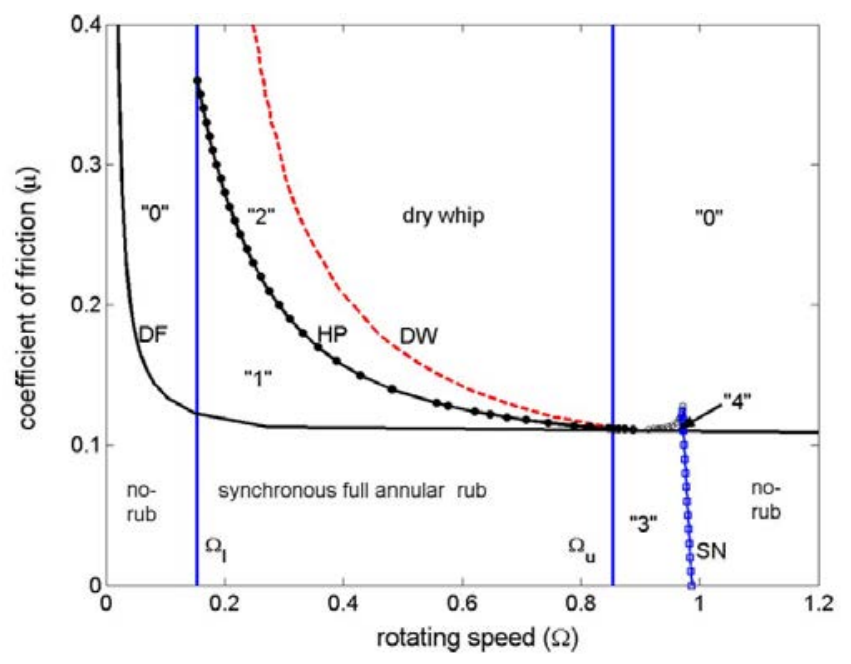

FIGURE 2. ROTOR RESPONSE CHARACTERISTICS IN THE PARAMETER PLANE OF $\Omega-\mu$ WITH $\beta=0.04, \xi=0.05, R_{0}=1.05$ AND $R_{\text {disk }}=20 R_{0}$, ACCORDING TO JIANG [10].

with the dimensionless variables: $X=\frac{x}{e}, \quad Y=\frac{y}{e}, \quad R_{0}=$ $\frac{r_{0}}{e}, \quad R_{\text {disk }}=\frac{r_{\text {disk }}}{e}, \quad V_{\text {rel }}=\frac{v_{\text {rel }}}{e}, \quad 2 \xi=\frac{c}{\sqrt{k_{b} m}}, \quad \beta=\frac{k_{s}}{k_{b}}, \quad \omega_{2}=$ $\sqrt{\frac{k_{b}}{m}}, \quad \Omega=\frac{\omega}{\omega_{2}}, \quad \omega_{b}=\frac{\omega_{w}}{\omega_{2}}, \quad R=\sqrt{X^{2}+Y^{2}}, \quad \Theta=$ $\left\{\begin{array}{ll}1 & \text { if } R \geq R_{0}, \\ 0 & \text { else. }\end{array}, \quad \tau=\omega_{2} t\right.$ where $x$ and $y$ represent the radial displacements along the $\mathrm{x}$ and $\mathrm{y}$ axes, respectively, $e$ is the mass eccentricity, $r_{0}$ is the initial clearance between the rotor and the stator, $r_{\text {disk }}$ is the disk radius, $v_{\text {rel }}$ is the relative velocity between the rotor and the stator, $c$ is the damping factor, $m$ is the rotor mass, $k_{s}$ and $k_{b}$ are the rotor and stator stiffnesses, respectively, $\omega$ is the rotor's rotational speed, and $\omega_{w}$ is the rotor's whirl frequency.

As seen in the equations of motion (8) and (9) above, the stator model is circular, rigid and fixed. There are no DOF associated with it. The contact is modelled by a penalty law using a dry friction Coulomb model.

Jiang analytically examines the existence of four possible types of steady-state rotor behaviour, resulting from imbalance and rotor-to-stator contact. These four types of behaviour are:

'no-rub motion' in which there is no contact between the rotor and the stator. The orbit is circular and the movement is periodic.

'full annular rub' in which the rotor remains in permanent contact with the stator. The orbit is also circular and the movement periodic.

'partial rub' in which the rotor intermittently comes into contact with the stator. The movement is quasi-periodic.

'backward whip' in which the rotor rolls (with slippage) on the inner surface of the stator. This movement is a selfexcited phenomenon and its frequency does not necessarily depend on the rotor's rotational speed. As a consequence, the movement is a priori quasi-periodic.

The stability of these four types of behaviour can be derived from the aforementioned dimensionless parameters. More specifically, Fig. 2 shows the rotor response characteristics in the $\Omega-\mu$ parameter plane. According to Jiang, the straight lines $\Omega_{l}$ and $\Omega_{u}$ indicate the rotational speed limits when the linear rotor eccentricity is equal to the initial clearance. Thus, the 'norub motion' can appear only at rotational speeds below $\Omega_{l}$ and above $\Omega_{u}$. The curves HP and SN correspond to the limits of 'full annular rub' motion. To the right of the line HP, 'full annular rub' becomes unstable and the movement becomes quasiperiodic (Hopf bifurcation). To the right of line SN, the 'full annular rub' no longer exists (saddle node bifurcation). The line DF denotes the presence of a backward whip response. The line DW indicates the limit beyond which backward whip is triggered by the imbalance force.

There are some regions in which several behaviours coexist. The regions labelled ' 0 ' are regions where no-rub motion and dry whip coexist. In the region labelled '1', both full annular rub and dry friction backward whirl are possible. The region labelled '2' shows the presence of partial rub and backward whirl. In '3', full annular rub and no-rub motions are simultaneously present. Finally, a small region '4' exhibits no-rub motion, full annular rub and backward whip.

The results shown in Fig. 2 have been confirmed via numerical simulations. Applying the same parameter values as those used in Fig. 2, the simulations were run with a fixed value for the friction coefficient $\mu$ and varying values of $\Omega$. Only one harmonic was retained for the HBM simulations. As the no-rub and full annular rub motions have circular orbits, one harmonic is sufficient to provide an exact representation of these movements. The pseudo arc length continuation algorithm was used for the parametric study with respect to $\Omega$, over the range from 0 to 1.2. In the case of the simulations based on the time marching technique, the solutions were calculated for discrete values of $\Omega$. The initial conditions used for the next value of $\Omega$ correspond to the final steady state conditions of the current simulation. This corresponds to an incremental continuation technique. For each value of $\Omega, 100$ rotor revolutions were simulated in order to attain the steady-state behaviour. Twenty-five additional revolutions were then simulated and saved for analysis. This procedure was performed twice for each value of $\mu$. In the first pass, the simulations were made with progressively greater values of $\Omega$, whereas in the second pass progressively smaller values of $\Omega$ were used. As numerical simulations (HBM or time integration) require the use of non dimensionless variables, the following parameters were set arbitrarily: $e=0.1 \mathrm{~m}, k_{s}=100 \mathrm{~N} \mathrm{~m}^{-1}, m=1$ $\mathrm{kg}$. The other parameters were derived. All of the simulations 


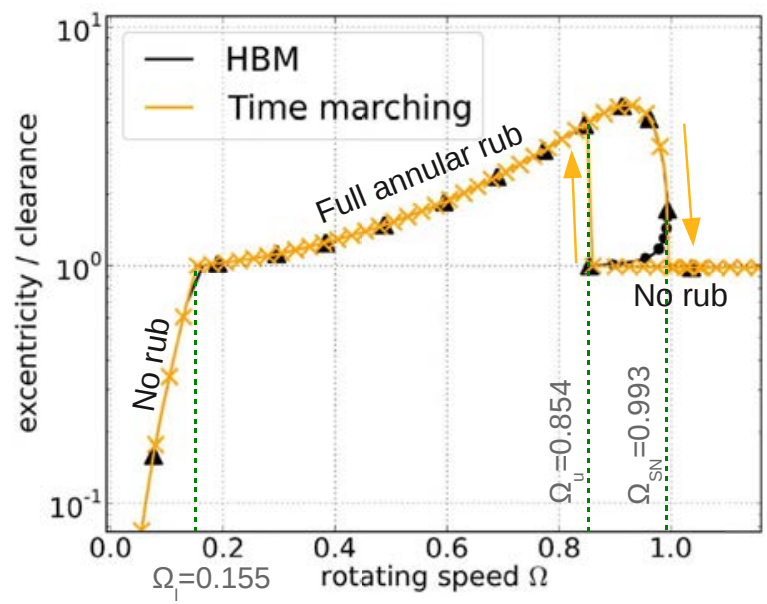

FIGURE 3. RESPONSE CURVE FOR $\mu=0.1$. HBM STABLE( $(\mathbf{\Delta})$, HBM UNSTABLE (.), TIME INTEGRATION $(\times)$.

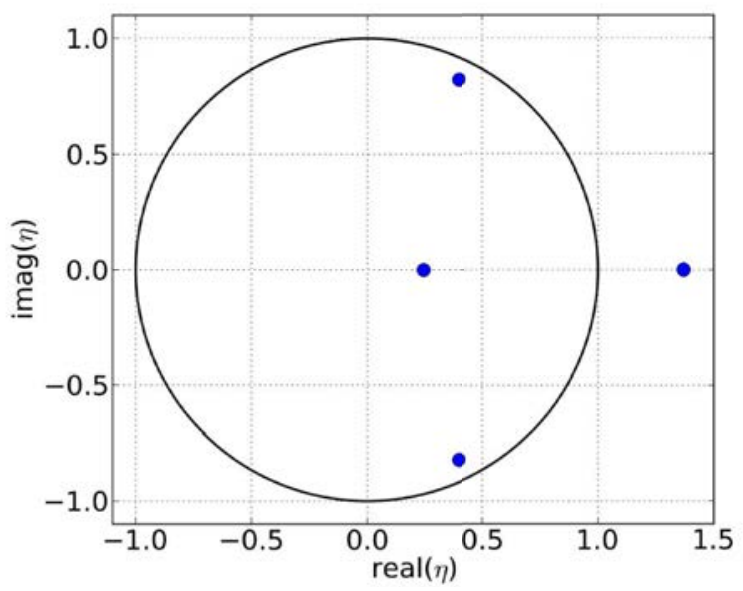

FIGURE 4. REAL AND IMAGINARY PARTS OF THE FLOQUET MULTIPLIERS IN THE UNSTABLE HBM SOLUTION AT $\Omega \approx 0.98$ (SADDLE NODE BIFURCATION)

were performed using the Code_Aster [17] and Scipy [18] environments. Although the simulations were run for many values of $\mu$, only the results obtained for $\mu=0.1$ and $\mu=0.2$, which are representative of all possible solutions, are presented in the following.

\section{Numerical Results}

Results for $\boldsymbol{\mu}=\mathbf{0 . 1}$. Fig. 3 shows the response curve for $\mu=0.1, \beta=0.04, \xi=0.05, R_{0}=1.05$ and $R_{\text {disk }}=20 R_{0}$

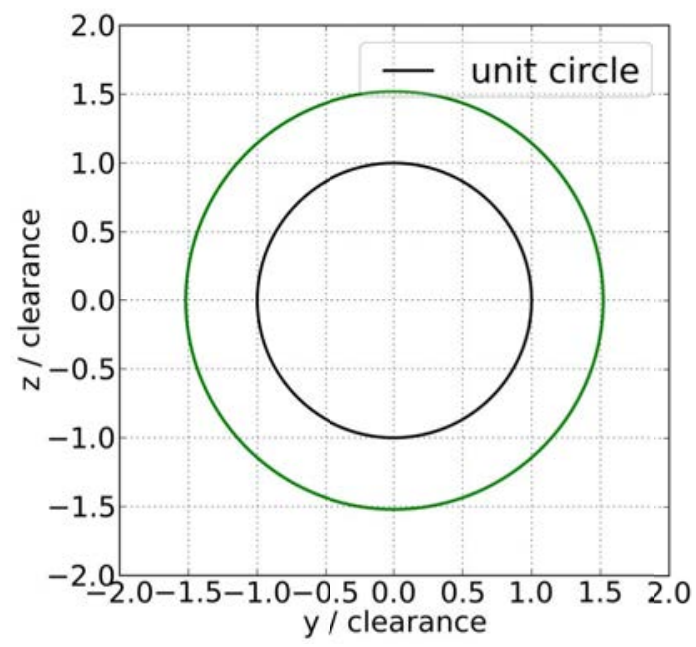

FIGURE 5. PERFECTLY CIRCULAR ORBIT OF THE ROTOR DURING FULL ANNULAR RUB AT $\Omega=0.5$.

calculated using HBM and time integration. The y-axis represents the rotor eccentricity normalized to the initial clearance, on a logarithmic scale, and the $\mathrm{x}$-axis corresponds to the normalized rotational speed $\Omega=\frac{\omega}{\omega_{2}}$. In this case, the time transient and HBM curves are very similar. As predicted by Jiang's analytical results (see Fig. 2), only no-rub motion and full annular rub are found for $\mu=0.1$. The transition between no-rub motion and full annular rub (which correspond to the $\Omega_{l}$ line in Fig. 2) occurs between approximately $\Omega=0.150$ and $\Omega=0.155$, which is highly consistent with the analytically determined value $\left(\Omega_{l}=0.1536\right)$. One can indeed see that the normalized rotor eccentricity is less than unity for $\Omega \leq 0.150$ and greater than unity for $\Omega \geq 0.155$. The $\Omega_{u}$ line is also correctly predicted by both HBM and the time integration methods $\left(\Omega_{u}=0.854\right)$. In addition, the region labelled ' 3 ' in Fig. 2 is also correctly predicted. Fig. 3 shows that no-rub motion and full annular rub are both possible for $0.850<\Omega<0.995$. The time integration method is characterised by a sudden 'drop' in eccentricity at $\Omega_{\mathrm{SN}} \approx 0.995$, corresponding to the 'run up' simulation, whereas a 'jump' occurs at $\Omega_{u} \approx 0.850$ during the 'run down' simulation. The HBM coupled to arc length continuation and stability computation is characterised by three possible solutions within this same range of rotational speeds, although only two of these are stable (passage through a saddle node bifurcation). The Floquet multipliers of the unstable HBM solution at $\Omega \approx 0.98$ are plotted in Fig. 4 . As the number of Floquet multipliers is equal to twice the number of degrees of freedom, four Floquet multipliers are visible. One of these has departed from the unit circle on the positive real axis, which corresponds here to a saddle node bifurcation. Although the HBM and time integration methods described here 


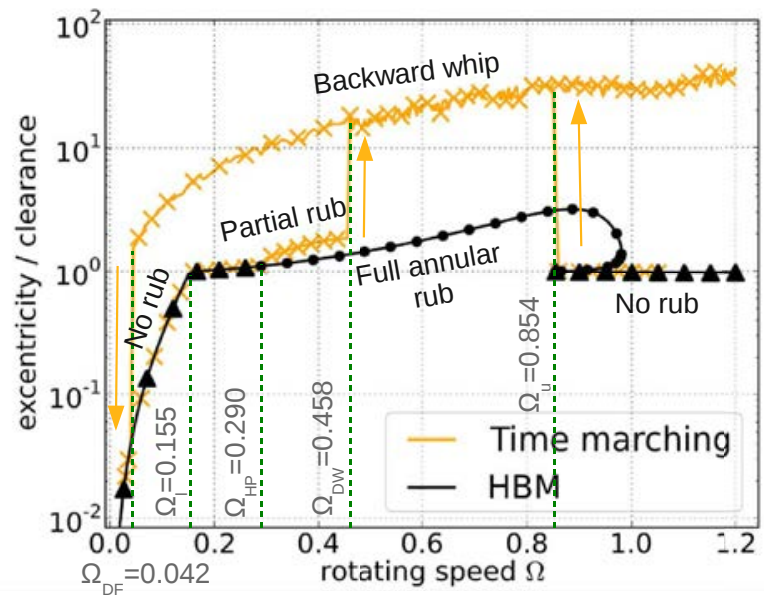

FIGURE 6. RESPONSE CURVE FOR $\mu=0.2$. HBM STABLE ( $\Delta$ ), HBM UNSTABLE (.), TIME INTEGRATION $(\times)$.

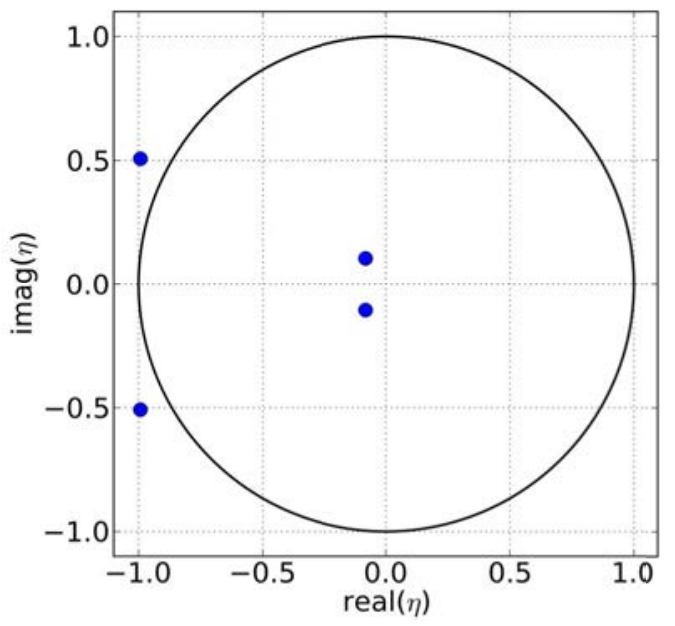

FIGURE 7. REAL AND IMAGINARY PARTS OF THE FLOQUET MULTIPLIERS IN THE UNSTABLE HBM SOLUTION AT $\Omega \approx 0.33$ (SECONDARY HOPF BIFURCATION).

provide extremely similar results, the HBM technique is approximately 500 times faster than the time integration method. The circular orbit of the rotor during full annular $\operatorname{rub}(\Omega=0.5)$ is shown in Fig. 5. Of course, the no-rub motion is also characterised by a perfectly circular orbit.

Results for $\boldsymbol{\mu}=\mathbf{0 . 2}$ The response curve for $\mu=0.2$ is shown in Fig. 6. Here, the HBM curve is very similar to that obtained for $\mu=0.1$. However, stability analysis reveals a loss

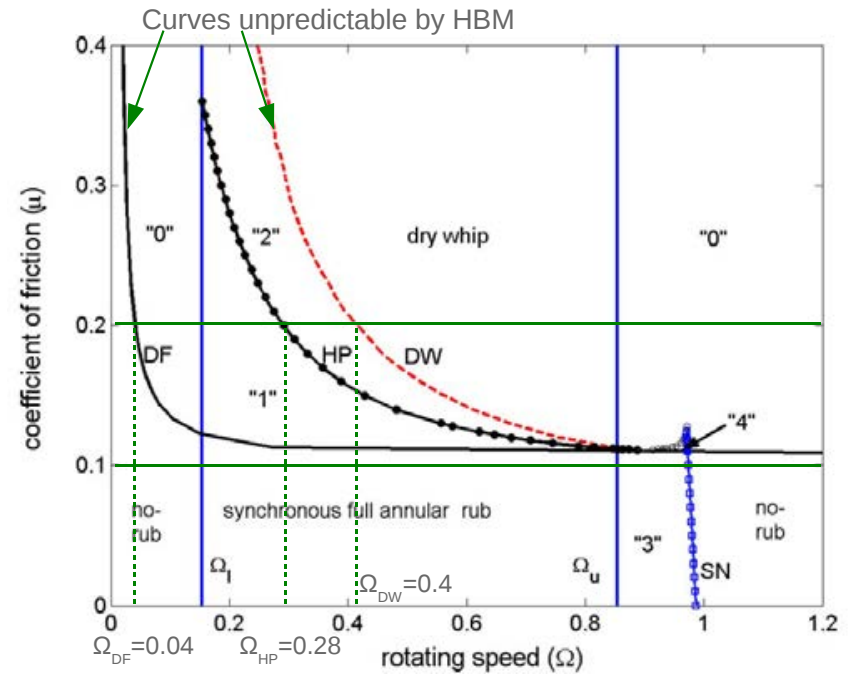

FIGURE 8. SAME AS FIG. 2 WITH NOTEWORTHY VALUES OF $\Omega$ HIGHLIGHTED FOR $\mu=0.1$ AND $\mu=0.2$ [10].

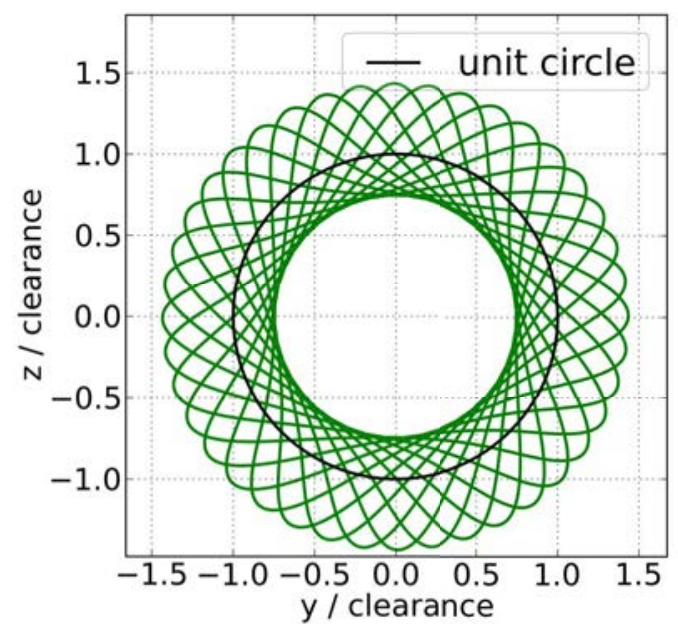

FIGURE 9. ROTOR ORBIT DURING PARTIAL RUB AT $\Omega=0.33$ (TIME TRANSIENT RESULT).

of stability beyond $\Omega_{\mathrm{HP}} \approx 0.289$. The Floquet multipliers of the unstable solution at $\Omega \approx 0.3$ can be seen Fig. 7 . A pair of Floquet multipliers has departed from the left-hand side of the unit circle, with a non-zero imaginary component indicating a Hopf bifurcation and a loss of stability. A Hopf bifurcation corresponds to the appearance of a second frequency in the real rotor response, and the initiation of a quasi-periodic regime. This means that although, theoretically, the periodic full annular rub response still exists beyond this point, it is in practice no longer possible to 


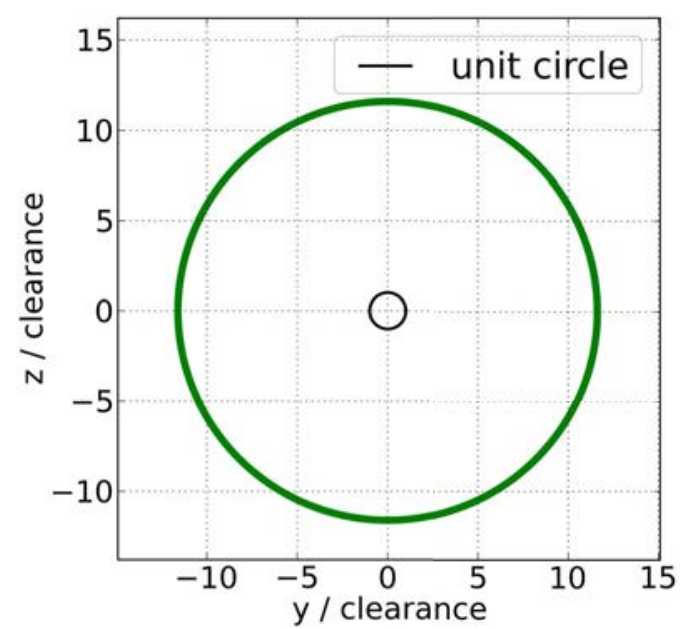

FIGURE 10. BACKWARD WHIP ORBIT AT $\Omega=0.33$. THE ROTOR ECCENTRICITY IS ONE ORDER OF MAGNITUDE GREATER THAN THE CLEARANCE (TIME TRANSIENT RESULT)

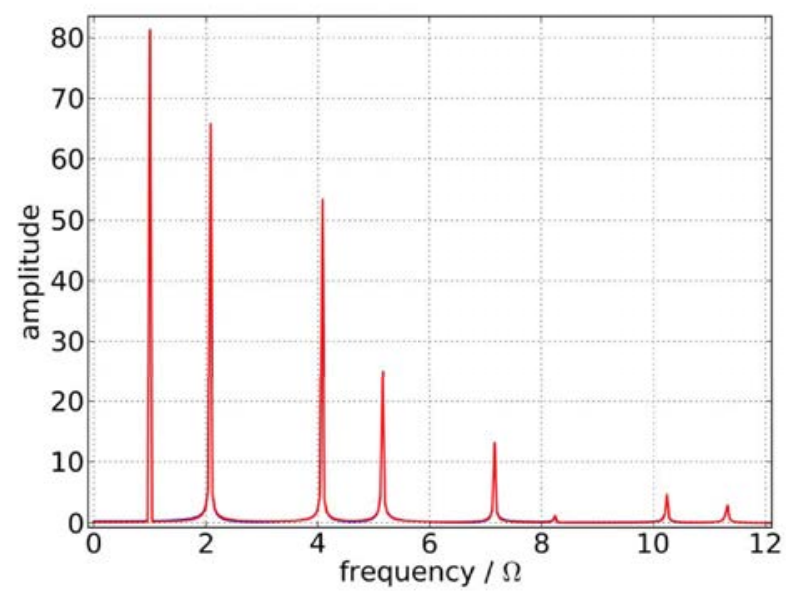

FIGURE 11. FAST FOURIER TRANSFORM OF THE NONLINEAR CONTACT FORCE SIGNAL DURING PARTIAL RUB AT $\Omega=$ 0.33. PEAKS AT LINEAR COMBINATIONS OF THE TWO INCOMMENSURABLE FREQUENCIES ARE VISIBLE.

achieve this response, due to its instability. The value of $\Omega_{\mathrm{HP}} \mathrm{ob}-$ tained with the HBM is very consistent with the value predicted by Jiang (see Fig. 8).

Conversely, the transient simulation results presented here are quite different to those previously observed. During the 'sweep up' simulation, the time transient results are initially sim-

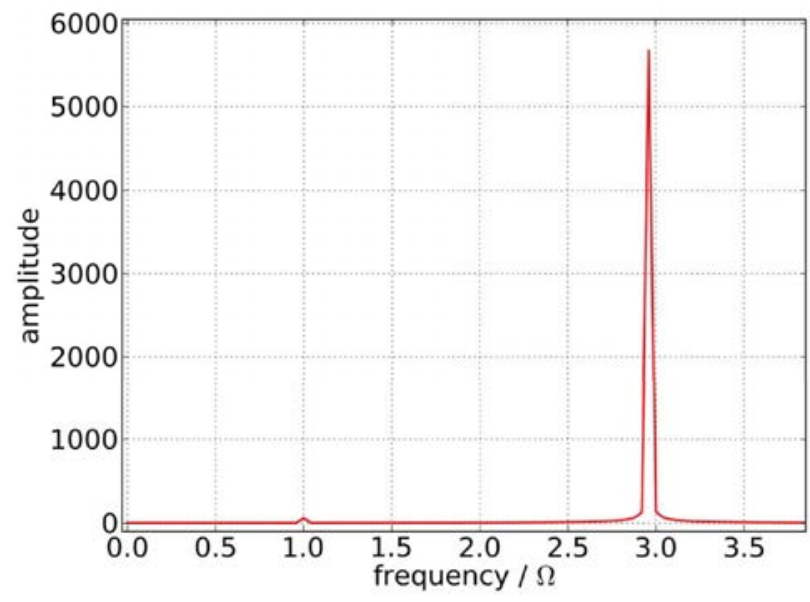

FIGURE 12. FAST FOURIER TRANSFORM OF THE NONLINEAR CONTACT FORCE SIGNAL DURING BACKWARD WHIP $\Omega=$ 0.33. ONLY TWO PEAKS CAN BE SEEN.

ilar to those observed for $\mu=0.1$. The no-rub motion is the first to be observed, and is very close to the HBM prediction. Then, beyond $\Omega_{l} \approx 0.15$, the full annular rub regime starts. Here again, the results are very close to those found with the HBM simulations. However, beyond $\Omega_{\mathrm{HP}} \approx 0.289$, the time transient line deviates from that predicted by the HBM, and the rotor motion becomes quasi-periodic. The rotor orbit computed using the time transient technique at $\Omega=0.33$ is shown Fig. 9. It can be seen that the rotor does not remain in permanent contact with the stator, which corresponds to the 'partial rub' motion predicted by Jiang. During this movement, the maximum rotor eccentricity is greater than that predicted during full annular rub. At $\Omega_{\mathrm{DW}} \approx 0.460$, it can be observed that the eccentricity suddenly increases by a factor of ten. Although slightly different from the value found by Jiang $(\approx 0.41)$, the latter value of $\Omega$ is coherent with this author's prediction. In this regime, referred to as backward dry whip, the rotor's orbit is almost circular, as shown in Fig. 10. The rotor can be seen to remain in the backward whip regime until the end of the sweep up.

A substantially different behaviour is observed during the sweep down. The rotor starts in the no-rub regime, until $\Omega_{u} \approx$ 0.86 , at which a sudden increase in eccentricity occurs, transferring the rotor motion to the backward whip regime. The rotor remains in this regime until a very small value of $\Omega_{\mathrm{DF}} \approx 0.045$ is reached, at which the eccentricity suddenly drops, leading to the no rub regime. This value of $\Omega_{\mathrm{DF}}$ is also in good agreement with Jiang's prediction (see Fig. 8).

From the preceding description, it can be seen that the HBM is able to predict only a portion of the time transient simulations. However, stability analysis can detect unstable solutions, such 
TABLE 1. $f_{n_{0}}$ AND $f_{n_{1}}$ CALCULATED FOR THE FIRST FOUR VALUES OF $n$ FOR THE PARTIAL RUB MOTION AT $\Omega=0.33$. HERE, $f_{1}=1 \times \Omega$ AND $f_{2} \approx 1.08 \times \Omega$.

\begin{tabular}{ccc}
\hline$n$ & $f_{n_{0}}(\times \Omega)$ & $f_{n_{1}}(\times \Omega)$ \\
\hline 0 & 1 & 2.08 \\
1 & 4.08 & 5.16 \\
2 & 7.16 & 8.24 \\
3 & 10.2 & 11.3 \\
\hline
\end{tabular}

that the DF curve can be determined in addition to the $\Omega_{l}$ and $\Omega_{u}$ lines. The DW and DF lines cannot be predicted by the HBM in its current form. Nonetheless, the HBM technique could be extended to quasi-periodic analysis $[19,20]$, theoretically allowing the partial rub and backward whip regimes to be predicted.

Transient simulations can provide valuable information concerning the nature of the quasi-periodic regimes. A fast Fourier transform of the nonlinear contact force, during partial rub at $\Omega=0.33$, is shown Fig. 11. By analyzing this diagram, it can be concluded that the peaks are present for linear combinations of two incommensurable frequencies. The lowest frequency (noted $\left.f_{1}\right)$ is naturally the rotational frequency of the rotor $(1 \times \Omega)$. The second frequency (noted $f_{2}$ ) cannot be accurately determined, since it is not commensurable with $f_{1}$. However, it can be approximated, for this value of $\Omega$, to approximately $1.08 \times \Omega$. Spectral analysis of the contact force during partial rub for several values of $\Omega$ show that, even though $f_{1}$ is naturally always equal to the rotating frequency $\left(f_{1}=1 \times \Omega\right)$, the second frequency $f_{2}$ is related to $f_{1}\left(f_{2}=f\left(f_{1}\right)\right)$. Although we noted that this second frequency $f_{2}$ decreases when $\Omega$ increases, no trivial relationship could be found relating them to each other. It was verified that, for every simulated partial rub movement, spectral peaks appeared in the contact force power spectrum, at linear combinations of the two incommensurable frequencies, as described by the following formulae:

$$
\begin{aligned}
& f_{n_{0}}=(2 n+1) f_{1}+(n+0) f_{2} \quad \text { for } n \in \mathbb{N} \\
& f_{n_{1}}=(2 n+1) f_{1}+(n+1) f_{2} \quad \text { for } n \in \mathbb{N}
\end{aligned}
$$

The peaks indeed occur in pairs, and decrease in amplitude with $n$ (see Fig. 11). The values of $f_{n_{0}}$ and $f_{n_{1}}$ are given in Table 1 for increasing values of $n$, (to be compared with Fig. 11).

The spectral analysis provided in Fig. 12 shows that the rotor movement has one main frequency during backward whip.
This predominant frequency does not correspond to the rotational speed of the rotor, but rather to the backward whip frequency. The rotational frequency of the rotor $f_{1}$ has a comparatively small amplitude, because the backward whip phenomenon is self-excited. Our computations show that the backward whip frequency has little to no dependence on $\Omega$. For $\Omega \leq 0.5$ the backward whip frequency increases slightly when $\Omega$ increases (from $0.75 \times \omega_{2}$ at $\Omega=0.045$ to $1 \times \omega_{2}$ at $\Omega=0.5$ ). For $\Omega \geq 0.5$ the backward whip frequency is constant and equal to $\omega_{2}$. This type of backward whip frequency behaviour is frequently reported in the literature, as for example in [12].

Other simulations were made for a large set of values of $\mu$. For each value tested, good agreement was found between the HBM, the time transient computations, and Jiang's prediction. Unfortunately, Jiang's predictions are limited to Jeffcott rotor models. Nonetheless, the HBM and time transient tools can be used for more complex models, such as finite element rotor models, with no need for any further developments.

\section{CONCLUSION}

The applicability of the Harmonic Balance Method (HBM), with pseudo arc-length continuation and stability assessment, to the computation of the steady-state behaviour of a rotor to stator contact problem, has been investigated. The HBM results have been compared with classical time marching solutions and analytical results [10]. The rotor model was that of a simple Jeffcott rotor, with a circular, rigid, static stator. It has been demonstrated that although the rotor is submitted to imbalance only, there are four possible kinds of solution: two of these are periodic solutions (no-rub motion and full annular rub), whereas the remaining two are quasi-periodic (partial rub and backward whip). The HBM is shown to be capable (at speeds orders of magnitude faster than those given by time marching analysis) of accurately predicting the two periodic solutions. The HBM can also predict the initiation of the quasi-periodic partial rub regime, which corresponds to a loss of stability of the periodic solution. However, the partial rub and backward whip motions cannot be described by means of the HBM in the presented form. An extension of this method to the treatment of quasi-periodic solutions is mandatory $[19,20]$, and is currently under study. Our detailed analysis of the partial rub and backward whip regimes, using time marching algorithms, has provided clues for the implementation of this extension.

\section{ACKNOWLEDGMENT}

This work was partially supported by the French National Agency (ANR) in the framework of its Technological Research COSINUS program (IRINA, project ANR 09 COSI 00801 IRINA) 


\section{REFERENCES}

[1] Edwards, S., Lees, A. W., and Friswell, M. I., 1999. "The influence of torsion on rotor/stator contact in rotating machinery". Journal of Sound and Vibration, 225(4), pp. 767 -778 .

[2] Feng, Z., and Zhang, X.-Z., 2002. "Rubbing phenomena in rotor-stator contact”. Chaos, Solitons \& Fractals, 14(2), pp. $257-267$.

[3] Popprath, S., and Ecker, H., 2007. "Nonlinear dynamics of a rotor contacting an elastically suspended stator". Journal of Sound and Vibration, 308(3-5), pp. 767 - 784. VibroImpact Systems.

[4] Han, Q., Zhang, Z., and Wen, B., 2008. "Periodic motions of a dual-disc rotor system with rub-impact at fixed limiter". Proceedings of the Institution of Mechanical Engineers, Part C: Journal of Mechanical Engineering Science, 222(10), Oct., pp. 1935-1946.

[5] An, X., Zhou, J., Xiang, X., Li, C., and Luo, Z., 2009. "Dynamic response of a rub-impact rotor system under axial thrust". Archive of Applied Mechanics, 79(11), Nov., pp. 1009-1018.

[6] Grapis, O., Tamuzs, V., Ohlson, N.-G., and Andersons, J., 2006. "Overcritical high-speed rotor systems, full annular rub and accident". Journal of Sound and Vibration, 290(35), pp. $910-927$.

[7] Braut, S., Zigulic, R., Skoblar, A., Stimac, G., Butkovic, M., and Jokic, M., 2007. "Dynamic analysis of the rotorstator contact due to blade loss". In 12th IFToMM World Congress, Besanon (France), June18-21, 2007.

[8] Qin, W., Chen, G., and Meng, G., 2004. "Nonlinear responses of a rub-impact overhung rotor". Chaos, Solitons \& Fractals, 19(5), pp. $1161-1172$.

[9] Jiang, J., and Ulbrich, H., 2001. "Stability analysis of sliding whirl in a nonlinear jeffcott rotor with cross-coupling stiffness coefficients". Nonlinear Dynamics, 24(3), Mar., pp. 269-283.

[10] Jiang, J., 2009. "Determination of the global responses characteristics of a piecewise smooth dynamical system with contact". Nonlinear Dynamics, 57(3), Aug., pp. 351361.

[11] Bently, D. E., Goldman, P., and Yu, J. J., 2002. "Full annular rub in mechanical seals, part ii: Analytical study". International Journal of Rotating Machinery, 8(5), pp. 329336.

[12] Childs, D. W., and Bhattacharya, A., 2007. "Prediction of dry-friction whirl and whip between a rotor and a stator". Journal of Vibration and Acoustics, 129(3), pp. 355-362.

[13] Sundararajan, P., and Noah, S. T., 1998. “An algorithm for response and stability of large order non-linear systems application to rotor systems". Journal of Sound and Vibration, 214(4), pp. $695-723$.

[14] Petrov, E. P., and Ewins, D. J., 2003. "Analytical formula- tion of friction interface elements for analysis of nonlinear multi-harmonic vibrations of bladed disks". Journal of Turbomachinery, 125(2), pp. 364-371.

[15] Lau, S. L., Cheung, Y. K., and Wu, S. Y., 1982. “A variable parameter incrementation method for dynamic instability of linear and nonlinear elastic systems". Journal of Applied Mechanics, 49(4), pp. 849-853.

[16] Cameron, T. M., and Griffin, J. H., 1989. “An alternating frequency/time domain method for calculating the steadystate response of nonlinear dynamic systems". Journal of Applied Mechanics, 56(1), pp. 149-154.

[17] EDF R\&D, 2001-. Code_aster: A general code for structural dynamics simulation under gnu gpl licence. http://www.code-aster.org.

[18] Jones, E., Oliphant, T., Pearu, et al., 2001-. Scipy: Open source scientific tools for Python. http://www.scipy.org/.

[19] Lau, S. L., Cheung, Y. K., and Wu, S. Y., 1983. "Incremental harmonic balance method with multiple time scales for aperiodic vibration of nonlinear systems". Journal of Applied Mechanics, 50(4a), pp. 871-876.

[20] Roques, S., 2006. "n-dimensional harmonic balance method extended to non-explicit nonlinearities". Revue Européenne de Mécanique Numérique, 15, pp. 269-280. 